\title{
Validação de uma tecnologia educativa: Manual para manejo clínico da amamentação na atenção primária
}

\author{
Validation of an educational technology: Manual for clinical management of breastfeeding in \\ primary care \\ Validación de una tecnología educativa: Manual para el manejo clínico de la lactancia materna en \\ atención primaria
}

Analécia Dâmaris da Silva Alexandre

ORCID: https://orcid.org/0000-0002-4912-3867

Universidade Federal do Maranhão, Brasil Universidade Estadual do Pará, Brasil E-mail: mestradocipe2019@gamail.com

José Antônio Cordero da Silva

ORCID: https://orcid.org/0000-0002-4403-5665 Universidade Estadual do Pará, Brasil E-mail: mestradocipe2019@gmail.com

Kecyani Lima dos Reis

ORCID: https://orcid.org/0000-0003-4168-2514 Faculdade de Ciências Médicas de Marabá, Brasil E-mail: tiakecy@hotamil.com

Mariseth Carvalho de Andrade ORCID: https://orcid.org/0000-0002-6257-5972 Universidade Estadual do Pará, Brasil

E-mail: marisethandrade1967@gmail.com

Gisele Rodrigues de Oliveira

ORCID: https://orcid.org/0000-0002-1229-0215 Universidade Estadual do Pará, Brasil E-mail: gisele.r.carvalho@bol.com.br

Jaqueline Miranda de Oliveira

ORCID: https://orcid.org/0000-0001-7066-4636 Universidade Estadual do Pará, Brasil E-mail: miranda.jaque01@gmail.com

Hugo Santana dos Santos Junior ORCID: https://orcid.org/0000-0002-8970-9897 Faculdade de Teologia, Piscicologia e Ciências, Gamaliel, Brasil E-mail: hugojuniorbs@bol.com.br

\begin{abstract}
Resumo
As evidências do impacto da amamentação vão muito além dos benefícios à saúde das mães e seus bebês. A Atenção Primária tem um papel fundamental no sentido de oferecer profissionais capacitados e atualizados para o incentivo dessa prática. O problema dessa pesquisa é: Como pode ser validado um manual para guiar o enfermeiro no manejo da amamentação na atenção primária. O objetivo foi validar um manual para manejo clínico da amamentação na atenção primária em Marabá. Trata-se de pesquisa aplicada de tecnologia educativa, de caráter experimental para validação do conteúdo de um manual para manejo clínico da amamentação a ser utilizado por enfermeiros da atenção primária. Foi utilizado o cálculo Alfa de Cronbach para investigar a consistência das respostas dos juízes através das variâncias entre as pontuações das perguntas apresentadas. Para determinar a validação de conteúdo do manual foi utilizado o IVC (índice de validação de conteúdo) para calcular as concordâncias entre as respostas dos juízes. O Manual para Manejo Clínico da Amamentação na Atenção Primária em Marabá foi estruturado em 16 capítulos. Na validação apresentou Alfa de Cronbach 0,86 com o objetivo de verificar a consistência do formulário respondido pelos juízes especialistas. O manual foi categorizado como validado no que concerne à relevância com variação de IVC de 0,91 tendo como índice de aprovação 96,9\%. O manual foi considerado validado para uso, sendo uma tecnologia de grande utilidade e aplicabilidade na prática clínica de assistência à dupla mãe - bebê, desde o pré-natal até o pós-parto.
\end{abstract}

Palavras-chave: Amamentação; Manejo clínico; Tecnologias em saúde; Validação de dados. 


\begin{abstract}
The evidence of the impact of breastfeeding goes far beyond the health benefits to mothers and their babies. Primary care plays a key role in providing trained and updated professionals to encourage this practice. The problem of this research is: How can a manual to guide nurses in the management of breastfeeding in primary care be validated? The objective was to validate a manual for clinical management of breastfeeding in primary care in Marabá. This is an applied experimental research of educational technology to validate the content of a manual for clinical management of breastfeeding to be used by nurses in primary care. The Cronbach's alpha calculation was used to investigate the consistency of the judges' responses through the variances between the scores of the questions presented. To determine the content validation of the manual, the CVI (content validation index) was used to calculate the concordances between the judges' responses. The Manual for Clinical Management of Breastfeeding in Primary Care in Marabá was structured in 16 chapters. At validation it presented Cronbach's alpha 0.86 with the objective of verifying the consistency of the form answered by the expert judges. The manual was categorized as validated in terms of relevance with a CVI variation of 0.91 , with an approval rate of $96.9 \%$. The manual was considered validated for use, being a technology of great usefulness and applicability in clinical practice of assistance to the mother-child pair, from prenatal to postpartum.
\end{abstract}

Keywords: Breastfeeding; Clinical management; Health technologies; Data validation.

\title{
Resumen:
}

Las pruebas del impacto de la lactancia materna van mucho más allá de los beneficios para la salud de las madres y sus bebés. La atención primaria desempeña un papel fundamental al proporcionar profesionales formados y actualizados para fomentar esta práctica. El problema de esta investigación es: ¿Cómo se puede validar un manual para orientar a las enfermeras en el manejo de la lactancia materna en atención primaria? El objetivo fue validar un manual para el manejo clínico de la lactancia materna en la atención primaria en Marabá. Se trata de una investigación aplicada de tecnología educativa de carácter experimental para validar el contenido de un manual de manejo clínico de la lactancia materna para ser utilizado por las enfermeras de atención primaria. El cálculo del alfa de Cronbach se utilizó para investigar la consistencia de las respuestas de los jueces a través de las varianzas entre las puntuaciones de las preguntas presentadas. Para determinar la validación del contenido del manual, se utilizó el IVC (índice de validación del contenido) para calcular las concordancias entre las respuestas de los jueces. El Manual de Manejo Clínico de la Lactancia Materna en la Atención Primaria de Marabá fue estructurado en 16 capítulos. En la validación, presentó el alfa de Cronbach 0,86 con el objetivo de verificar la consistencia del formulario respondido por los jueces expertos. El manual se clasificó como validado en cuanto a su pertinencia, con una variación del IVC de 0,91 y una tasa de aprobación del 96,9\%. El manual se consideró validado para su uso, siendo una tecnología de gran utilidad y aplicabilidad en la práctica clínica de asistencia al dúo madre-bebé, desde el prenatal hasta el posparto.

Palabras clave: Lactancia materna; Gestión clínica; Tecnologías sanitarias; Validación de datos.

\section{Introdução}

As evidências do impacto da amamentação vão muito além dos benefícios à saúde das mães e seus bebês. Contribuem com a economia dos países, desde a redução dos gastos com tratamentos de doenças, que são silenciadas por componentes do leite materno, até o aumento da renda gerada por adultos, que quando crianças foram amamentadas por mais tempo e desenvolveram uma inteligência elevada (Fp Hartwig, 2018).

A amamentação contribui para o alcance de pelo menos cinco objetivos para o desenvolvimento sustentável até 2030 : nutrição, redução da pobreza, educação, redução de sobrepeso e obesidade, câncer de mama e diabetes, além de favorecer o crescimento econômico inclusivo (Victoria et al, 2016).

Na década de 70, após a Organização Mundial de Saúde (OMS) e o Fundo das Nações Unidas para a Infância (UNICEF) realizarem uma iniciativa mundial para sensibilizar a sociedade quanto aos benefícios do aleitamento materno, verificou-se uma movimentação de muitos países implementando mudanças em suas políticas públicas de incentivo a amamentação exclusiva (Perilo et.al, 2019).

No contexto brasileiro, pode-se destacar algumas políticas de promoção, proteção e apoio à amamentação, como por exemplo: Iniciativa Hospital Amigo da Criança (IHAC), Método Canguru, Rede Brasileira de Bancos de Leite Humano (RBLH), Estratégia Amamenta e Alimenta Brasil, além da Norma brasileira de comercialização de alimentos para lactentes e crianças de primeira infância (NBCAL). Outras Políticas brasileiras que também têm sido fundamentais no incentivo ao aleitamento materno exclusivo são a Política Nacional de Atenção Integral à Saúde da Criança (PNAISC), a Rede Cegonha, a 
Política Nacional de Atenção Básica (PNAB), A Política Nacional de Promoção da Saúde (PNPS), a Política Nacional de Alimentação e Nutrição (PNAN) e a Política Nacional de Educação Permanente (Brasil, 2013).

Resultados Preliminares dos Indicadores de Aleitamento Materno no Brasil, disponíveis no Estudo Nacional de Alimentação e Nutrição Infantil (ENANI 2019), apontam que aleitamento materno exclusivo entre menores de quatro e de seis meses, e o aleitamento materno continuado aos 12 meses e entre menores de 24 meses, apresentaram uma evolução positiva e significativa nos últimos 34 anos, analisando os dados por macrorregião. Essa pesquisa mapeou o aleitamento materno no país, e avaliou 14.505 crianças menores de cinco anos, entre fevereiro de 2019 e março de 2020, demonstrou que $53 \%$ das crianças menores de um ano continua sendo amamentada; entre os bebês menores de seis meses o índice encontrado foi de 45,7\%; e entre os menores de 4 meses, 60\% (Enani, 2019).

A amamentação por vezes tem sido vista como um processo que é estabelecido instintivamente e até mesmo automaticamente. Essa visão fortalece as verdades absolutas que a sociedade consolidou através da cultura e outras ferramentas sociais, como por exemplo, acreditar que todas as mulheres conseguem amamentar exclusivamente, e que todas são capazes de produzir tudo o que seu bebê precisa. Essas máximas desconsideram a individualidade e história de vida de cada mulher, se afastando do cuidado integral tão almejado na assistência durante o ciclo gravídico puerperal, além de invisibilizar as dificuldades que podem surgir no processo de estabelecimento de Amamentação de cada dupla mãe - bebê; o que, consequentemente impede também a prevenção e solução dessas dificuldades (Mendes et. al, 2019).

Apesar de todas as políticas públicas implementadas e resultados de aumento na prevalência do aleitamento materno exclusivo, o desmame precoce ainda é uma preocupação para a saúde coletiva brasileira, por motivos relatados pelas mães como: ingurgitamento mamário, produção de leite insuficiente, mastite, fissuras nos mamilos; intercorrências geralmente relacionadas à pega e posicionamento incorretos, ausência de rede de apoio, falta de orientação e até mesmo apoio adequado dos profissionais que prestam assistência à dupla mãe- bebê durante essas dificuldades (Margotti, 2016).

Em Marabá, o Banco de Leite Humano foi criado em 2012, mesmo ano da implantação da Rede Cegonha no município. Por condução do Banco de Leite Humano local, anualmente acontece a Campanha para incentivo à Doação de Leite Materno. Nas Unidades Básicas de Saúde, onde as mulheres são acompanhadas durante o pré-natal e o pós-parto, são realizadas também as atividades com orientações de incentivo e proteção do aleitamento materno exclusivo. E os protocolos efetivados nesses atendimentos são os recomendados pelo Ministério da Saúde.

As mulheres cadastradas no pré-natal, recebem orientações referente ao aleitamento materno exclusivo durante a consulta de enfermagem, seja ela na gestação ou mesmo no pós-parto. Assim, esse profissional no âmbito da atenção primária local, é um agente que atua, através da assistência direta à dupla mãe- bebê, ou mesmo no encaminhamento as demandas necessárias ao acompanhamento com outros profissionais da rede de saúde.

O aleitamento materno é uma responsabilidade compartilhada por toda a sociedade, e não apenas pela mulher. Nesse contexto, a Atenção Primária tem um papel fundamental no sentido de oferecer profissionais capacitados e atualizados para o incentivo dessa prática, ainda na gravidez e o manejo clínico da amamentação no pós-parto, desde os primeiros dias de vida do bebê (Sawaya et al, 2019).

Neste sentido, os profissionais que acompanham mais de perto a amamentação, necessitam de atualização e instrumentos específicos que produzam práticas de promoção ao aleitamento materno e assistência às intercorrências mais frequentes, a fim de que sejam realmente capazes de manejar corretamente a amamentação em qualquer período, o que contribui significativamente no aumento da prevalência do aleitamento materno exclusivo (Bonilha et.al, 2010).

Diante desse quadro, o enfermeiro da atenção primária que oferece assistência à gestante e a dupla mãe-bebê, necessita de atualização constante e instrumentos norteadores que lhe deem fundamentação estratégica para orientar e intervir de forma adequada, quando necessário; cuidando também para que seja respeitado o desejo da mãe, além de garantir o 
acolhimento em todas as situações, aliado ao exercício da empatia, com o objetivo de estabelecer uma relação de confiança, aumentando o nível de adesão da mãe ao plano terapêutico proposto (Queiroz, 2003; Brasil, 2015).

Considerando ainda que, o Parecer do Conselho Federal de Enfermagem (COFEN n ${ }^{\circ}$ 18/2016/CTAS) afirma que o Enfermeiro é um profissional liberal, capacitado na sua formação superior e respaldado legalmente para realizar consultoria em amamentação e puerpério atendendo as Políticas Públicas emanadas pelo Ministério da Saúde, bem como possuem amparo legal para solicitação de exames laboratoriais a fim de desenvolverem a assistência plena de Enfermagem de forma ética e com competência técnica.

Nessa perspectiva, a atualização dos conhecimentos e utilização das tecnologias que possam auxiliar no processo assistencial se faz cada vez mais necessária, visto que proporciona ao profissional de saúde um suporte rápido e extremamente rico cientificamente, podendo prover a assistência de forma mais segura e padronizada (Santos, 2018).

A elaboração de materiais didáticos de apoio e suporte científico tem crescido nos últimos anos, tecnologias de consulta rápida, prática e barata estão cada vez mais presentes no cenário da assistência em saúde. A criação de manuais contribui de forma ímpar para esse processo de atualização dos meios de educação continuada e auxílio dos profissionais, apresentando uma gama de benefícios e aplicações no dia a dia de trabalho das equipes de saúde (Leite et al, 2014).

Considerando isso, o problema dessa pesquisa é: Como pode ser elaborado e validado um manual para guiar o enfermeiro no incentivo da amamentação na atenção primária, tanto durante o pré-natal como no pós-parto?

E o objetivo é elaborar e validar um manual para promoção do aleitamento materno exclusivo durante o pré-natal e o manejo clínico da amamentação no pós-parto na atenção primária em Marabá.

\section{Metodologia}

Trata-se de pesquisa aplicada de tecnologia educativa, com abordagem quantitativa, analítica, descritiva de caráter experimental que objetivou a validação do conteúdo de um manual para manejo clínico da amamentação a ser utilizado por enfermeiros da atenção primária.

Para Silva Medeiros et al (2015, p.5) os estudos quantitativos desenvolvidos através de analises em relação a validade de conteúdos e tecnologias educativas são de extrema importância para comunidade acadêmica em geral, pois proporciona dados palpáveis quanto a aplicabilidade de validade cientifica de um determinado conteúdo ou ferramenta. Ressalta que o processo de validação dos instrumentos expressa segurança, aplicabilidade, confiabilidade e validade.

A metodologia quantitativa com abordagem analítica descritiva proporciona apresentar os dados coletados em uma determinada amostra ou levantamento epidemiológico e estático de forma a estruturar uma comparação entre os dados obtidos e com isso analisar e interpretar dos dados coletados, de forma palpável, fiel e contribui para a visualização dos quantitativos relevantes ao tema da pesquisa em questão. Através dos dados sendo possível a tabulação e organização dos resultados obtidos no processo metodológico e estruturação de gráficos e tabelas (Taquette \& Borges 2021).

\subsection{Aspectos éticos, local do estudo e período de coleta de dados}

Os aspectos éticos foram respeitados em todas as etapas da pesquisa, em conformidade com a Convenção de Helsinki, além de estar alinhada com as Resoluções n. ${ }^{\circ} 466 / 12$ e 510/16 que tratam das normas para pesquisas envolvendo seres humanos. Com isso, por se tratar de pesquisa de cunho quantitativo, descritivo analítico, sem intervenção, coleta de dados ou pesquisa com seres humanos, não foi necessário à submissão e autorização pelo Comitê de Ética em Pesquisa - CEP. Sendo assim, os dados sigilosos pertinentes ao processo de análise quantitativa não foram disponibilizados. 
O local de realização do estudo foi na cidade Marabá, região sudeste do estado do Pará, na macrorregião de Carajás. O período de realização de coleta de dados do estudo foi de 15 dias, desde o dia 15 de março até dia 30 do mesmo mês no ano de 2021.

\subsection{Critérios de inclusão e exclusão - juízes}

O manual será utilizado por profissionais que atuam nas Unidades Básicas de Saúde -(UBS) do município de MarabáPa. Este município faz parte da mesorregião sudeste do estado do Pará, e apresenta 25 UBS, com 14 localizadas na zona urbana e 11 na zona rural. As Unidades Básicas do município de Marabá são compostas por equipes de Estratégia de Saúde da Família (ESF) e Estratégia de Agentes Comunitários de Saúde (EACS), que atuam de acordo com os programas do ministério da saúde (hipertensão e diabetes, tabagismo, tuberculose, hanseníase, saúde da mulher, saúde da criança, saúde do adolescente, saúde do homem, imunizações, saúde do idoso e saúde na escola).

Como critérios de inclusão foi utilizado ser o profissional com titulação de enfermeiro, no mínimo especialista na área de ginecologia e obstetrícia, neonatologia, pediatria ou saúde da família, com exercício profissional de pelo menos dois anos e local de atuação na atenção primária e/ou secundária na região do sudeste do Pará. Os critérios de exclusão estabelecidos foram profissionais de outras áreas da saúde que não fosse enfermeiro, e não tenha especialização de ginecologia e obstetrícia, ou saúde da família, e que não atue na atenção primária ou secundária da região sudeste do Pará. Foram excluídos e substituídos, aqueles que não devolveram as respostas ao questionário de validação em tempo hábil para finalização da pesquisa.

Os participantes da pesquisa foram profissionais enfermeiros especialistas e mestres, em ginecologia e obstetrícia, pediatria, saúde da família ou neonatologia, atuantes na Atenção Primária em Marabá e/ou região sudeste do Pará. A Tabela 1 apresenta as características dos juízes que participaram da coleta de dados. A tabela descreve as características dos avaliadores que participaram da validação do manual. Os dados estão separados em cinco tópicos relevantes, de acordo o sexo, a faixa etária, o tempo de formação dos profissionais, os títulos de especialistas e qual especialidade.

Tabela 1 - Perfil dos Juízes selecionados para coleta de dados da validação.

\begin{tabular}{|c|c|c|c|}
\hline \multicolumn{2}{|c|}{ Perfil dos juízes } & Frequência & \% (n = 9) \\
\hline Sexo & Feminino & 7 & $78 \%$ \\
& Masculino & 2 & $22 \%$ \\
\hline Faixa etária & 30 a 40 & 2 & $22 \%$ \\
& 40 a 50 & 7 & $78 \%$ \\
\hline Tempo de formação & 5 a 10 & 2 & $22 \%$ \\
& 10 a 19 & 3 & $33 \%$ \\
\hline Titulo de especialistas & 20 a 29 & 9 & $100 \%$ \\
\hline \multirow{3}{*}{ Qual especialidade } & SIM & & $67 \%$ \\
& NÃO & 6 & $11 \%$ \\
& Ginecologia e & 1 & $22 \%$ \\
\hline
\end{tabular}

Fonte: Autores (2021).

O processo de validação do manual contou com dados obtidos através das respostas de os nove profissionais de saúde seguindo os critérios de inclusão. As faixas etárias dos juízes foram de 30 a 40 , e 40 a 50 anos de idade. Foram 7 profissionais do sexo feminino e 2 profissionais do sexo masculino. Em relação á formação acadêmica, um total de 8 especialistas e 1 participante com mestrado profissional. Quanto aos anos de atuação, os profissionais descreveram os períodos entre 5 e 10, 10 
a 19, e 20 a 29 anos de exercício profissional. O local de trabalho informado refere a tenção primaria em saúde do município de Marabá.

Os profissionais que atuam diretamente no manejo clínico da amamentação na atenção primária são os enfermeiros, realizando acolhimento, avaliação da situação clínica e implementando intervenções personalizadas a cada dupla mãe- bebê, além de encaminhar a outros profissionais da equipe da unidade básica ou ao serviço especializado, conforme a necessidade identificada durante o acompanhamento.

\subsection{Estrutura do manual e questionário validado}

O manual foi elaborado de acordo com as diretrizes e manuais do Ministério da Saúde (Cadernos da Atenção Básica), Conselho Federal de Enfermagem (COFEN - PARECER COFEN No 18/2016), Federação Brasileira das Sociedades de Ginecologia e Obstetrícia (FEBRASGO) e artigos científicos, priorizando os que apresentem evidências de resultados exitosos no manejo clínico da amamentação, e mesmo estudos que apontem as estratégias necessárias para a melhoria dos índices de aleitamento materno exclusivo até os 6 meses, além de respeitar normas e rotinas do Sistema Único de Saúde (SUS), por se tratar de atenção primária de saúde relacionada à saúde da mulher e da criança.

O Manual para Manejo Clínico da Amamentação na Atenção Primária em Marabá foi estruturado em 16 capítulos, que tratam das principais intercorrências relacionadas às dificuldades em amamentar e o frequente desmame precoce, sejam elas diretamente ligadas à mãe, ao bebê, ou a díade, simultaneamente. Nesse cenário, traz como diferencial a apresentação em quadros onde são descritas as principais características, definição e as intervenções sugeridas, além do fluxograma de encaminhamento das Intercorrências Emocionais e Frênulo Lingual na Rede de Atenção Primária em Marabá. E ainda, o quadro de intervenção do Dhysphoric Milk Ejection Reflex D-MER, ou Disforia no Reflexo de Ejeção do Leite, intercorrência ainda pouco conhecida e recentemente veiculada na comunidade científica.

O instrumento de coleta de dados no processo de validação foi um questionário de validado e já utilizado para validação de manuais, livros, guias e cartilhas. Tal ferramenta é composta por 17 perguntas divididas em 3 partes, onde cada parte representa um assunto a ser avaliado no questionário. Com isso, a primeira parte é composta por perguntas em relação aos objetivos do manual. A segunda parte do questionário é formada de perguntas sobre a estrutura do manual, a organização e apresentação dos conteúdos que compõem o manual. Por fim, a terceira parte é composta de perguntas que tratam da relevância da temática do manual. Para cada pergunta do questionário as alternativas de escolha foram estruturadas em 3 respostas: $1^{\circ}$ inadequado; $2^{\circ}$ parcialmente adequado e $3^{\circ}$ totalmente adequado. Comtemplando assim, uma alternativa negativa, uma positiva e uma representando certa neutralidade.

A plataforma de construção do questionário na versão digital foi o Google Formulários, tendo a fase da coleta através de envio do questionário usando as ferramentas digitais Whatsapp e E-mail dos juízes especialistas. Os profissionais que concordaram em participar da pesquisa assinaram o termo de consentimento livre e esclarecido (TCLE) em forma digital em duas vias de igual teor, sendo uma cópia para o pesquisador e outra para o especialista. Foi apresentado ao profissional os objetivos da pesquisa, sendo que no momento da explicação dos termos do TCLE foi frisado os direitos do participante do estudo, sendo que a desistência a qualquer momento, é um direito do profissional, se assim o desejasse, ressaltando que a desistência não lhe traria qualquer prejuízo. Finalmente, foi informado que a identidade do sujeito seria sempre preservada tanto durante a condução do estudo como em publicações posteriores.

\subsection{Análise estatística}

A etapa de análise estatística para sua execução foi dividido em duas partes, sendo a primeira a avaliação da consistência e qualidade do questionário de validação para coletada de dados utilizando o cálculo do Alfa de Cronbach 
objetivando verificar o formulário respondido pelos juízes especialistas e analisando a consistência do instrumento através de avalição da variância entre os juízes e as pontuações dadas a cada pergunta. A Tabela 2 demonstra os intervalos do cálculo do Alfa de Cronbach e seus respectivos significados.

Tabela 2 - classificação e intervalos do Alfa de Cronbach.

\begin{tabular}{|c|c|}
\hline Valor de Alfa & Classificação \\
\hline 0,91 ou mais & Excelente \\
\hline $0,90 \nmid 0,81$ & Bom \\
\hline $0,81+0,71$ & Aceitável \\
\hline $0,71+0,61$ & Questionável \\
\hline $0,61+0,51$ & Pobre \\
\hline Menor que 0,51 & Inaceitável \\
\hline
\end{tabular}

Fonte: Freitas, A. L. P., \& Silva, V. B. D. (2014). (Adaptação autores, 2021)

Os intervalos do Alfa de Cronbach são analisados através dos dados obtidos com o cálculo de variância entre os pontos positivos e almejados. Com isso, considerando os intervalos de maior consistência os valores entre 0,71 e 1 .

A execução da avaliação da consistência usando o Alfa de Cronbach para verificar a consistências dos questionários validados utilizados em coleta de dados é de extrema relevância cientifica, pois proporciona verificar vários âmbitos e partes do questionário que possam interferir na coleta. Entender e verificar questões duplicadas e com duplo sentido ou questões tendenciosas a má interpretação, avaliando toda a estrutura do questionário e sua qualidade fundamental validando o questionário para utilização como instrumento de coleta para avaliação de manuais, guias, livros e instrumentos tecnológicos (Almeida, et al, Santos, Costa, 2010).

Na segunda parte da fase estatística foi utilizado o Índice de Validade do Conteúdo (IVC) tendo esse que ser igual ou superior a 0,78. O IVC mede a concordância entre as opiniões dos especialistas, avaliando os objetivos, estrutura e apresentação, e relevância do manual, dando uma pontuação positiva para cada pergunta na validação. Os especialistas no assunto ressaltam que o instrumento tecnológico seja ele manual, livro ou cartilha deverá receber no mínimo 078 no IVC, contudo alguns autores ressaltam que esse valor seja de no mínimo 0,80 ou superior (Polit, \& Beck, 2011)

O índice foi calculado por meio da soma de concordância dos itens que foram marcados como "2" e " 3 " pelos especialistas, dividido pelo número total de respostas. Para avaliar o protocolo de forma geral foi utilizada uma das formas de cálculo recomendada por Polit e Beck (2004), na qual o somatório de todos os IVC calculados separadamente é dividido pelo número de itens do instrumento, devendo o valor ser também superior a 0,78 para ser considerado validado. A Figura 1 demonstra o IVC e como é o cálculo e sua interpretação.

Figura 1 - Cálculo do índice de Validade de Conteúdo (IVC).

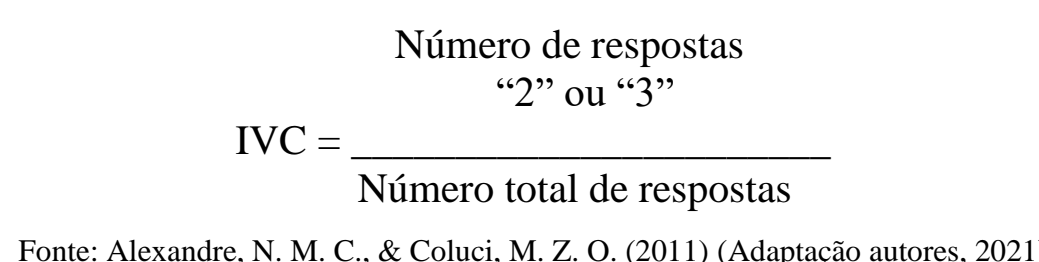

Os elementos amostrais foram tratados em um banco de dados processados no software Microsoft Office Excel 2013. Organizados e apresentados em tabelas. 


\section{Resultados e Discussão}

Os resultados foram divididos em duas partes, sendo a primeira sobre a análise da consistência do questionário pelo Alfa de Cronbach onde se obteve o resultado do cálculo de 0,86. Os resultados obtidos através do Alfa de Cronbach demostram que a consistência adequada para coleta de dados usando o instrumento, de acordo com a classificação do Alfa o questionário recebeu a análise de "bom".

$\mathrm{Na}$ segunda etapa dos resultados temos a parte que realizou o cálculo e análise do IVC, onde foi avaliado a concordância entre as perguntas e as respostas dadas pelos juízes especialistas que participaram da validação, apresentando e determinando a validade do conteúdo do manual. A Tabela 3, 4 e 5 demonstra a organização das perguntas de acordo com cada critério e o grau de concordância dos juízes para cada pergunta.

Tabela 3 - Concordância das respostas dos juízes quanto aos objetivos do manual.

\begin{tabular}{|l|c|}
\hline \multicolumn{1}{|c|}{ Avaliação do Manual: OBJETIVOS } & Concordância \\
\hline $\begin{array}{l}\text { Coerentes do ponto de vista da promoção e proteção do aleitamento } \\
\text { materno na atenção primária. }\end{array}$ & $92,6 \%$ \\
\hline $\begin{array}{l}\text { Atende aos objetivos da atenção primária que trabalha com manejo clínico } \\
\text { da amamentação. }\end{array}$ & $92,6 \%$ \\
\hline Pode circular no meio científico na área da enfermagem obstétrica. & $96,3 \%$ \\
\hline $\begin{array}{l}\text { Coerentes com as necessidades do profissional na atenção primária nas } \\
\text { consultas de acompanhamento da amamentação. }\end{array}$ & $100,0 \%$ \\
\hline
\end{tabular}

Fonte: Autores (2021).

Os resultados obtidos sobre os objetivos do manual e o nível de concordância dos juízes especialistas demonstram que para a pergunta 1 sobre se os dados são coerentes quanto a promoção do aleitamento materno foi de 92,6\% de concordância; 92,6\% de concordância para pergunta 2 sobre os objetivos do manual para o assunto de manejo da amamentação na atenção primária; 96,3\% para pergunta 3 sobre a possibilidade do manual circular como ferramenta cientifica no meio profissional, e $100 \%$ de concordância na pergunta 4 sobre os objetivos do manual em relação as necessidades do profissional quanto ao manejo do aleitamento materno.

A Tabela 4 demonstra os resultados sobre a estrutura e apresentação dos conteúdos do manual de manejo da amamentação. 
Tabela 4 - Concordância das respostas dos juízes quanto à estrutura e apresentação do manual.

\begin{tabular}{|l|c|}
\hline \multicolumn{1}{|c|}{ Avaliação do Manual: ESTRUTURA e APRESENTAÇÃO } & Concordância \\
\hline As figuras são expressivas e suficientes. & $85,2 \%$ \\
\hline As informações estão bem estruturadas em concordância e ortografia. & $88,9 \%$ \\
\hline Informações da capa, contracapa e apresentação são coerentes. & $96,3 \%$ \\
\hline $\begin{array}{l}\text { Suporte de orientação aos enfermeiros nas consultas de acompanhamento da } \\
\text { amamentação. }\end{array}$ & $100,0 \%$ \\
\hline As mensagens estão apresentadas de maneira clara e objetiva. & $100,0 \%$ \\
\hline As informações apresentadas estão cientificamente corretas. & $100,0 \%$ \\
\hline Apresenta sequência lógica do conteúdo proposto. & $100,0 \%$ \\
\hline O tamanho do título e dos tópicos está adequado. & $100,0 \%$ \\
\hline O número de páginas está adequado. & $100,0 \%$ \\
\hline
\end{tabular}

Fonte: Autores (2021).

Em relação ao nível de concordância dos juízes sobre as perguntas com a temática da estrutura, organização e apresentação das informações do manual, 82,2\% dos especialistas concordaram sobre a pergunta 1 sobre a expressividade das figuras no manual; na pergunta $288,9 \%$ concordaram sobre a estrutura e apresentação dos dados no manual e sua ortografia; 96,3\% concordaram sobre a pergunta 3 que diz respeito a capa, contra capa e estrutura das informações; $100 \%$ de concordância na pergunta 4 sobre a utilização do manual como suporte consultivo no assunta amamentação; na quinta pergunta sobre o clareza e objetividade do manual os juízes concordaram em 100\%; na sexta pergunta os juízes julgaram o critério cientifico das informações e concordaram em 100\%; sobre a sequência lógica das informações os juízes concordaram em 100\%; na pergunta 9 que indaga sobre o tamanho dos títulos e tópicos os juízes concordaram em $100 \%$ e na pergunta 10, onde se trata sobre o número de páginas do manual a concordância foi de $100 \%$.

Tabela 5 demonstra os dados dos resultados obtidos em relação às perguntas sobre a relevância do manual de amamentação e o nível de concordância dos juízes especialistas em cada pergunta.

Tabela 5 - Concordância das respostas dos juízes sobre a relevância do manual.

\begin{tabular}{|l|c|}
\hline \multicolumn{1}{|c|}{ Avaliação do Manual: RELEVÂNCIA } & Concordância \\
\hline Os temas retratam aspectos-chave que devem ser reforçados. & $96,3 \%$ \\
\hline $\begin{array}{l}\text { Propõe adquirir mais conhecimento quanto às condutas adequadas nas consultas de } \\
\text { manejo da amamentação. }\end{array}$ & $100,0 \%$ \\
\hline $\begin{array}{l}\text { Aborda assuntos necessários a serem oferecidos na assistência de enfermagem á dupla } \\
\text { mãe bebê no período da Amamentação. }\end{array}$ & $100,0 \%$ \\
\hline $\begin{array}{l}\text { Adequado para ser usado por enfermeiros durante as consultas de acompanhamento da } \\
\text { amamentação na atenção primária. }\end{array}$ & $100,0 \%$ \\
\hline
\end{tabular}

Fonte: Autores (2021).

A Tabela 5 demonstra os resultados de concordância sobre a relevância do manual de manejo clínico da amamentação onde na pergunta 1 sobre a relevância dos aspectos chaves do manual os juízes concordaram com 96,3\%; na segunda pergunta sobre o acumulo de conhecimentos sobre a amamentação e sua relevância no manejo clinico os especialistas concordaram em $100 \%$; na pergunta 3 sobre o manual ser um instrumento que ofereça assuntos essenciais sobre o atendimento de enfermagem a 
dupla mãe bebê na amamentação os juízes concordaram em 100\%; na pergunta 4 sobre o manual ser adequado para ser usado por enfermeiros durante os atendimentos no aleitamento materno na atenção básica em saúde os juízes concordaram em $100 \%$.

A Tabela 6 contém os resultados gerais sobre o cálculo e análise do Alfa de Cronbach sobre a validade e consistência entre as variâncias do questionário e da avalição geral do índice de validade de conteúdo - IVC em relação ao grau de concordância dos juízes especialista na validação do manual de manejo clínico da amamentação.

Tabela 6 - Resultados gerais do Alfa de Cronbach, IVC e aprovação em porcentagem.

\begin{tabular}{|c|c|c|c|c|}
\hline $\begin{array}{c}\text { Critérios para validação do } \\
\text { manual }\end{array}$ & $\begin{array}{c}\text { Pontuação } \\
\text { Recebida }\end{array}$ & $\begin{array}{c}\% \\
\text { Aprovação }\end{array}$ & IVC & Cronbach \\
\hline Objetivos & 103 & $95,4 \%$ & 0,861 & 0,833 \\
\hline Estrutura e Apresentação & 235 & $96,7 \%$ & 0,914 & 0,887 \\
\hline Relevância & 107 & $99,1 \%$ & 0,972 & 0,833 \\
\hline Geral & 445 & $96,9 \%$ & 0,915 & 0,866 \\
\hline
\end{tabular}

Fonte: Autores (2021).

Os dados gerais demonstram que na realização do cálculo do Alfa de Cronbach para avalição do questionário o resultado foi de 0,86 apresentando validade suficiente. Em relação ao índice de validação de conteúdo - IVC o resultado geral foi de 0,91 e a aprovação geral do manual de manejo clínico da amamentação na atenção primária foi de 96,9\%.

A importância do enfermeiro como educador no contexto do aleitamento materno na atenção primária deve ser valorizada. Tal profissional desenvolve papel fundamental para efetivação de práticas qualificadas de assistência em saúde no âmbito da atenção básica. Com isso, o suporte de instrumentos e materiais que auxiliem esses profissionais no decorrer do trabalho é de extrema necessidade (Da Silva, et al, 2019).

A elaboração de tecnologias educativas em várias áreas de atuação em saúde contribui para uniformizar as orientações e condutas dos profissionais que prestam cuidados assistenciais de forma direta e indireta em vários âmbitos do Sistema Único de Saúde (SUS), mais acentuadamente na atenção primária em saúde, pois representa a porta de entrada para o serviço de saúde (Figueiredo et al, 2019).

O desenvolvimento de cartilhas, guias, manuais e ferramentas de acesso rápido e prático que implicam diretamente na educação continuada das equipes de saúde e dos enfermeiros assistenciais são de extrema valia (Da Silva, et al, 2019).

A atenção primária desenvolve atividades essenciais para o funcionamento do SUS no Brasil, executando ações de prevenção, promoção, proteção e recuperação da saúde. Para a efetivação integral e resolutiva as tecnologias educacionais em saúde no âmbito da atenção básica proporcionam melhor qualificação profissional, padronização da assistência, sem esquecerse de respeitar e considerar a singularidade de cada paciente. Tais instrumentos e tecnologias proporcionam o aumento da adesão do público alvo às boas práticas profissionais (Figueiredo et al, 2019).

A atualização profissional e instrumentos específicos contribuem para o aumento na prevalência do aleitamento materno exclusivo. Uma boa assistência promove melhores indicadores de saúde e consecutivamente maior impacto social frente às intercorrências na amamentação (Bonilha et al, 2010).

\section{Conclusão}

O Manual Manejo Clínico da Amamentação na Atenção Primária em Marabá, desenvolvido para enfermeiros para suporte no manejo das principais intercorrências relacionadas à amamentação no âmbito da atenção básica em saúde, foi considerado validado para uso, sendo uma tecnologia de grande utilidade e aplicabilidade na prática clínica de assistência à dupla mãe - bebê, desde o pré-natal até o pós-parto. 
A elaboração de materiais de suporte para profissionais de saúde atuantes na assistência é de extrema relevância e devem ser encorajados. Esses instrumentos transmitem capacitação e com isso maior segurança na atenção frente ao manejo clínico da amamentação e suas peculiaridades.

Ações de educação em saúde através da elaboração de instrumentos técnicos-científicos com embasamento atualizado e aprofundado fortalecem a prática assistencial da equipe assistencial atuante na atenção, em especial ao enfermeiro por ser um dos profissionais da equipe multidisciplinar com maior contato frente aos cuidados em relação à amamentação.

\section{Referências}

Bonilha, A. L. D. L., Schmalfuss, J. M., Moretto, V. L., Lipinski, J. M., \& Porciuncula, M. B. (2010). Capacitação participativa de pré-natalistas para a promoção do aleitamento materno. Revista brasileira de enfermagem, 63(5), 811-816.

Brasil. Ministério da Saúde. Portaria n 1920, de 5 de setembro de 2013. Institui a Estratégia Nacional para promoção do aleitamento materno e alimentação complementar saudável no Sistema único de Saúde (SUS) - Estratégia Amamenta e Alimenta Brasil. 2013.

Da Silva, A. X., Martins, G. F. R., Cavalcanti, M. D., de França, P. C. G., de Oliveira, A., Júnior, S., \& de Araújo Gomes, J. (2019). Assistência de enfermagem no aleitamento materno exclusivo: uma revisão integrativa. Brazilian Journal of Health Review, 2(2), 989-1004.

Figueiredo, S. V., Moreira, T. M. M., Mota, C. S., Oliveira, R. S. D., \& Gomes, I. L. V. (2019). Elaboração e validação de caderneta de orientação em saúde para familiares de crianças com doença falciforme. Escola Anna Nery, 23(1).

Sawaya, A. L., Peliano, A. M., Albuquerque, M. P. D., \& Domene, S. M. Á. (2019). A família e o direito humano à alimentação adequada e saudável. Estudos Avançados, 33(97), 361-382.

Hartwig, F. P. (2018). Aspectos genéticos e epigenéticos da amamentação.

Leite, K. N. S. (2014). A utilização das Tecnologias da Informação e Comunicação pelos docentes de enfermagem e as dificuldades no processo de ensinoaprendizagem.

Margotti, E., \& Mattiello, R. (2016). Fatores de risco para o desmame precoce. Rev Rene, 17(4), 537-544.

Mendes, S. C., Lobo, I. K. V., Sousa, S. Q. D., \& Vianna, R. P. D. T. (2019). Fatores relacionados com uma menor duração total do aleitamento materno. Ciência \& Saúde Coletiva, 24, 1821-1829.

Perilo, T. V. C. Tratado do especialista em cuidado materno-infantil com enfoque em amamentação/ Tatíana VargasCastro Perilo. - Belo Horizonte Mame Bem. 2019. 436p: 210x280mm.

Polit, D. F., Hungler, B. P., \& Beck, C. T. (2004). Avaliação da mensuração e da qualidade dos dados. Fundamentos da pesquisa em enfermagem: métodos, avaliação e utilização. Porto Alegre (RS): Artmed.

Santos, L. K. B. D. A. (2018). O uso das tecnologias digitais para o ensino em hemoterapia: construção e validação de um material didático para um curso a distância (Master's thesis, Brasil).

UFRJ. Universidade Federal do Rio de Janeiro. Estudo Nacional de Alimentação e Nutrição Infantil - ENANI-2019: Resultados preliminares - Indicadores de aleitamento materno no Brasil. UFRJ: Rio de Janeiro, 2020. 9 p. Modo de acesso: https://enani.nutricao.ufrj.br/index.php/relatorios/ 1. Estudo Nacional de Alimentação e Nutrição Infantil. 2. Aleitamento materno.

Victora, C. G., Barros, A. J., França, G. V., Bahl, R., Rollins, N. C., Horton, S., \& Walker, N. (2016). Amamentação no século 21: epidemiologia, mecanismos, e efeitos ao longo da vida. Epidemiol Serv Saúde, 25(1), 1-24.

Taquette, S. R., \& Borges, L. (2021). Pesquisa qualitativa para todos. Editora Vozes.

Queiroz, M. V. O. (2003). Rede de significados da educação em saúde: prática dos profissionais no cuidado da criança.

Da Silva Medeiros, R. K., Júnior, M. A. F., Pinto, D. P. D. S. R., Vitor, A. F., Santos, V. E. P., \& Barichello, E. (2015). Modelo de validação de conteúdo de Pasquali nas pesquisas em Enfermagem. Revista de Enfermagem Referência, 4(4), 127-135.

Freitas, A. L. P., \& Silva, V. B. D. (2014). Avaliação e classificação de instituições de ensino médio: um estudo exploratório. Educação e Pesquisa, 40(1), 2947.

Almeida, D., Santos, M. A. R. D., \& Costa, A. F. B. (2010). Aplicação do coeficiente alfa de Cronbach nos resultados de um questionário para avaliação de desempenho da saúde pública. XXX Encontro Nacional de Engenharia de Produção, 15, 1-12.

Alexandre, N. M. C., \& Coluci, M. Z. O. (2011). Validade de conteúdo nos processos de construção e adaptação de instrumentos de medidas. Ciência \& Saúde Coletiva, 16, 3061-3068.

Polit, D. F., \& Beck, C. T. (2011). Fundamentos de pesquisa em enfermagem: avaliação de evidências para a prática da enfermagem. Artmed Editora. 